\title{
Pesque-solte: pesca repetitiva, variáveis hematológicas e parasitismo no peixe híbrido tambacu
}

\author{
Daniela Nomura Varandas(1), Maurício Laterça Martins( ${ }^{(2)}$, Flávio Ruas de Moraes ${ }^{(3)}$, Fabrício Menezes Ramos ${ }^{(4)}$, \\ Rudã Fernandes Brandão Santos ${ }^{(5)}$ e Rodrigo Yudi Fujimoto(6)
}

\begin{abstract}
(1)Nutreco Brasil Nutrição Animal, Rua Álvaro Santana Ferreira, no 221, CEP 14887-386 Jaboticabal, SP. E-mail: daniela.nomura@gmail. com ${ }^{(2)}$ Universidade Federal de Santa Catarina, Centro de Ciências Agrárias, Departamento de Aquicultura, Rodovia Admar Gonzaga, no 1.346, Itacorubi, CEP 88034-001 Florianópolis, SC. E-mail: demlaterca@cca.ufsc.br (3)Universidade Estadual Paulista Júlio de Mesquita Filho (Unesp), Faculdade de Ciências Agrárias e Veterinárias, Departamento de Patologia Veterinária, Via de Acesso Prof. Paulo Donato Castellane, s/no, CEP 14884-900 Jaboticabal, SP. E-mail: fruasmoraes@gmail.com (4)Universidade Federal do Pará, Núcleo de Ciências Agrárias e Desenvolvimento Rural, Programa de Pós-Graduação em Ciência Animal, Campus Universitário do Guamá, Rua Augusto Corrêa, no 1, CEP 66075-110 Belém, PA. E-mail: fabriciomramos@gmail.com ${ }^{(5)}$ Unesp, Centro de Aquicultura, Laboratório de Patologia de Organismos Aquáticos. E-mail: ruda_fernandes@hotmail.com ${ }^{(6)}$ Embrapa Tabuleiros Costeiros, Avenida Beira Mar, no 3.250, Caixa Postal 44, CEP 49025-040 Aracaju, SE. E-mail: rodrigo.fujimoto@embrapa.br
\end{abstract}

Resumo - O objetivo deste trabalho foi avaliar a resposta hematológica e parasitológica de tambacus (Colossoma macropomum x Piaractus mesopotamicus) submetidos ao estresse de captura e a diferentes densidades de estocagem, em sistema de pesque-solte. Foram utilizados 210 peixes com peso médio inicial de $785,33 \pm 152,02 \mathrm{~g}$ e comprimento total médio de $34,43 \pm 2,21 \mathrm{~cm}$, mantidos em viveiros escavados e divididos em três grupos: sem pesca e baixa densidade (G1), com pesca e baixa densidade (G2), e com pesca e alta densidade (G3). Não houve diferença significativa entre os valores médios da concentração de hemoglobina, do número de eritrócitos, da contagem diferencial de leucócitos e da glicose. Os peixes do grupo G3 apresentaram número maior de parasitos e trombócitos, e menor ganho de peso e hematócrito. A atividade de pesque-solte, aliada à alta densidade de estocagem, pode prejudicar o equilíbrio orgânico e o desempenho zootécnico, o que favorece a parasitose.

Termos para indexação: Colossoma macropomum, Piaractus mesopotamicus, densidade de cultivo, parasitos, pesca esportiva.

\section{Catch-and-release: repetitive fishing, hematological variables and parasitism in the tambacu hybrid fish}

\begin{abstract}
The objective of this work was to evaluate the hematological and parasitological responses of tambacu (Colossoma macropomum x Piaractus mesopotamicus) subjected to catch-and-release stress and to different stocking densities in a catch-and-release system. Two hundred and ten fish with mean initial weight of $785.33 \pm 152.02 \mathrm{~g}$ and mean total length of $34.43 \pm 2.21 \mathrm{~cm}$ were maintained in ponds and divided into three groups: without fishing and low density (G1), with fishing and low density (G2), and with fishing and high density (G3). No significant differences were observed between the mean values for hemoglobin concentration, erythrocyte number, differential counting of leukocytes, and glucose. The fish from the G3 group showed higher parasite and thrombocyte numbers, and lower weight gain and hematocrit. The activity of catch-and-release, associated with high stocking density, can disrupt organic balance and animal performance, favoring parasitosis.

Index terms: Colossoma macropomum, Piaractus mesopotamicus, stocking density, parasites, sport fishing.
\end{abstract}

\section{Introdução}

O crescente número de adeptos da pesca esportiva estimula atividades como a do sistema de pesque-solte, em que os peixes são capturados com caniço e anzol, permanecem fora da água por alguns minutos, são manuseados e, em seguida, soltos. Essas ações constituem-se em estímulos estressantes, que levam o organismo a responder de forma não específica frente a situações que ameaçam desequilibrar a sua homeostase (Barton, 2002).

O indivíduo estimulado responde com hipersecreção de catecolaminas e cortisol, o que aumenta a mobilização e a utilização de reservas energéticas, e interfere no equilíbrio hidromineral, ao suprimir os mecanismos de defesa, alterar o leucograma, induzir hiperglicemia e determinar contração esplênica com lançamento de eritrócitos na circulação (Oba et al., 2009). 
O sistema de pesque-solte utiliza altas densidades de estocagem, e os viveiros tornam-se excessivamente eutrofizados, com redução da concentração de oxigênio dissolvido e aumento dos níveis de amônia. A alta densidade estimula maior competição entre os indivíduos e reduz o aproveitamento dos nutrientes (Papoutsoglou et al., 1998), o que causa prejuízo ao crescimento (Iwama et al., 2004). Já a deterioração do ambiente aquático, compromete o equilíbrio da relação parasito-hospedeiro-ambiente, o que favorece a proliferação de parasitas (Georgiadis et al., 2001; Marengoni et al., 2009).

Além da densidade e da carga parasitária, os peixes podem detectar a presença de predadores, a qual é comunicada aos coespecíficos por meio químico, para possibilitar a fuga (Jordão \& Volpato, 2000). Esse mesmo fenômeno poderia ocorrer em peixes fisgados, pois ocorrem lesões na pele semelhantes às obtidas no processo de predação, com a liberação de substâncias de alarme que estimulam os coespecíficos (Volpato, 2000; Wisenden \& Stacey, 2005; Wisenden \& Chivers, 2006), o que, por hipótese, poderia se constituir em estresse.

O tambacu, híbrido entre fêmeas de tambaqui [Colossoma macropomum (Cuvier, 1818)] e machos de pacu [Piaractus mesopotamicus (Holmberg, 1887)], destaca-se, nos pesqueiros, por apresentar rusticidade, valor comercial e carne saborosa, além de rápido crescimento e maior tolerância a baixas temperaturas que seus parentais (Gonçalves et al., 2010).

O objetivo deste trabalho foi avaliar a resposta hematológica e parasitológica de tambacus submetidos ao estresse de captura e a diferentes densidades de estocagem, em sistema de pesque-solte.

\section{Material e Métodos}

O experimento foi realizado no Centro de Aquicultura da Universidade Estadual Paulista Júlio de Mesquita Filho, Jaboticabal, SP, em 2001. Foram utilizados 210 tambacus com peso médio inicial de $785,33 \pm 152,02 \mathrm{~g}$ e comprimento total médio de $34,43 \pm 2,21 \mathrm{~cm}$, mantidos em viveiros com capacidade para $50 \mathrm{~m}^{3}$ de água, com fluxo contínuo.

Os peixes passaram por um período de adaptação de uma semana e foram distribuídos ao acaso, em três grupos: sem pesca e baixa densidade de estocagem $\left(0,709 \mathrm{~kg} \mathrm{~m}^{-2} ; \mathrm{n}=45\right)$; com pesca e baixa densidade $\left(1,058 \mathrm{~kg} \mathrm{~m}^{-2} ; \mathrm{n}=67\right)$; e com pesca e alta densidade $\left(1,561 \mathrm{~kg} \mathrm{~m}^{-2} ; \mathrm{n}=99\right)$.

Todos os animais foram marcados com miçangas coloridas, em que cada cor representava um número, de 1 a 210, o que permitiu a identificação individual quando pescados. Para marcação, os peixes foram anestesiados em solução de benzocaína $\left(1 \mathrm{~g}^{10} \mathrm{~L}^{-1}\right)$ (Gomes et al., 2001), e as miçangas foram fixadas por linha de pesca no dorso, na altura da nadadeira dorsal. Utilizou-se solução de álcool iodado a 10\% como antisséptico.

A ração utilizada continha $22 \%$ de proteína bruta (10\% de farinha de peixe, $38 \%$ de milho, $17,5 \%$ de farinha de soja, $16 \%$ de farinha de arroz, $16,5 \%$ de farinha de trigo, $1 \%$ de premix e $1 \%$ de óleo de soja) e foi fornecida quatro dias por semana (segunda a quinta), pela manhã e à tarde, sendo suspensa na sexta, no sábado e no domingo. O período de pesca ocorreu nos finais de semana (sexta a domingo), das $8 \mathrm{~h}$ às $12 \mathrm{~h}$ e das $14 \mathrm{~h}$ às $18 \mathrm{~h}$, com um pescador (escolhido aleatoriamente) no grupo 2 e outro no grupo 3. Foram utilizados anzóis com farpa, varas e linhas de $0,30 \mathrm{~mm}$ de diâmetro, com suporte de até 4,53 kg (10 libras). A isca foi feita com ração triturada e umedecida. Uma vez fisgados, os peixes foram mantidos na água até a exaustão e, em seguida, foram recolhidos, medidos, pesados, identificados e devolvidos aos viveiros. Esses últimos procedimentos duravam $5 \mathrm{~min}$ no total. Ao final do experimento, foram contabilizados 12 finais de semana consecutivos de pesca.

No último dia, sete peixes de cada grupo foram anestesiados em solução aquosa de benzocaína (1 g $\left.10 \mathrm{~L}^{-1}\right)$, para amostragem de sangue por punção da veia caudal, tendo-se utilizado agulhas e seringas heparinizadas. Uma parte da alíquota destinouse a extensões sanguíneas coradas com Giemsa/ May-Grunwald, para contagem diferencial de leucócitos e trombócitos. Neste caso, os trombócitos foram quantificados, em percentagem, juntamente com os leucócitos, por se tratarem de células envolvidas na resposta de defesa do organismo (Martins et al., 2006; Bozzo et al., 2007; Tavares-Dias et al., 2007). Outra alíquota foi utilizada para determinação do hematócrito. O restante foi armazenado em frascos de vidro no gelo, para determinação da glicemia e contagem total de eritrócitos em contador automático Celm, modelo CC510 (Celm - Cia. Equipadora de Laboratórios Modernos, Barueri, SP).

Pesq. agropec. bras., Brasília, v.48, n.8, p.1058-1063, ago. 2013 DOI: 10.1590/S0100-204X2013000800035 
Após a punção sanguínea, foram sacrificados 20 tambacus de cada grupo por comoção cerebral, para colheita de parasitos branquiais (Tavares-Dias et al., 2001a).

O ganho de peso e a intensidade de pesca foram calculados com as fórmulas:

Ganho de peso (individual) $=$ peso final - peso inicial

Intensidade de pesca $=$ número de peixes fisgados/ número de peixes no viveiro.

A temperatura, o oxigênio dissolvido, o $\mathrm{pH}$ e a condutividade elétrica da água foram medidos diariamente às $8 \mathrm{~h}$ e às $17 \mathrm{~h}$ com peagâmetro YSI 63 e oxímetro YSI 55 (YSI Incorporated, Yellow Springs, OH, EUA). A temperatura foi medida com termômetro comum de mercúrio. A amônia e o nitrito foram determinados duas vezes por semana nos dois períodos, segundo o método de Koroleff (1976) e Mackereth et al. (1978).

Os resultados foram submetidos à análise de variância, e quando significativas, as médias foram comparadas pelo teste de Tukey, a 5\% de probabilidade. Cada peixe foi considerado uma repetição.

\section{Resultados e Discussão}

A qualidade de água manteve-se dentro dos padrões estabelecidos para criação de peixes (Kubitza, 2011). Não houve diferença significativa entre os grupos de pesque e solte para as variáveis analisadas (Tabela 1).

Em relação à pesca, não foi observada diferença significativa quanto à intensidade de pesca entre os grupos 2 e 3 , e o estímulo de captura produzido não diferiu significativamente. No total, os peixes foram pescados 284 vezes - 103 no grupo 2 e 181 no grupo 3.

Dois peixes foram pescados cinco vezes e 15 deles quatro vezes no período de três meses. Alguns peixes foram pescados duas vezes no mesmo dia, com intervalo de poucas horas. Diferentemente do relatado por Volpato (2000), nos tambacus, o estímulo da pesca parece não ter estabelecido qualquer tipo de comunicação entre coespecíficos, nem inibido o acesso do peixe à isca ou ao anzol repetidamente.

O grupo 3 apresentou menor ganho de peso, possivelmente em razão da alta densidade e da pesca (Tabela 2), ou seja, foi o grupo que sofreu estímulos estressantes mais intensos.

Em condições de estresse, ocorre redução no crescimento somático e no ganho de peso, e os níveis de catecolaminas e cortisol se elevam como resposta ao estímulo, para consumo energético, gliconeogênese e lipólise (Oba et al., 2009). O crescimento também é prejudicado pelo menor aproveitamento dos nutrientes da ração (Papoutsoglou et al., 1998), e o estímulo da captura causa debilitação, o que pode resultar em taxas de mortalidade de até 89\% (Cooke \& Suski, 2005). Assim, no presente trabalho, a alta densidade e a pesca provavelmente foram responsáveis pelo menor ganho de peso dos peixes do grupo 3, enquanto os grupos $1 \mathrm{e}$ 2 não apresentaram diferença significativa (Tabela 2). Portanto, o prejuízo no ganho de peso não pode ser atribuído somente à pesca, pois a alta densidade também tem papel importante.

Ao analisar o efeito da captura com anzol em Argyrosomus hololepidotus (Lacépède, 1801), Broadhurst \& Barker (2000) observaram elevação nas concentrações de cortisol $30 \mathrm{~min}$ depois da captura, as quais retornaram aos níveis iniciais após 2 horas. Contrariamente ao observado no presente trabalho, estes autores não verificaram diferença no ganho de peso e no comprimento entre animais controle e capturados com anzol.

O exame parasitológico mostrou infestação branquial por Anacanthorus penilabiatus (Boeger, 1995) significativamente maior no grupo 3 , em comparação aos outros dois grupos (Tabela 2). Esses resultados se devem, além da pesca, à menor qualidade da água como resultado da elevada densidade de estocagem. A primeira favorece a proliferação dos parasitos, e a última resulta em maior susceptibilidade

Tabela 1. Valores médios dos parâmetros aquáticos dos viveiros dos grupos 1 (sem pesca e baixa densidade), 2 (com pesca e baixa densidade) e 3 (com pesca e alta densidade).

\begin{tabular}{lccccc}
\hline Parâmetros & $\begin{array}{c}\mathrm{O}_{2} \text { dissolvido } \\
\left(\mathrm{mg} \mathrm{L}^{-1}\right)\end{array}$ & $\begin{array}{c}\text { Amônia total } \\
\left(\mu \mathrm{g} \mathrm{L}^{-1}\right)\end{array}$ & $\begin{array}{c}\text { Temperatura } \\
\left({ }^{\circ} \mathrm{C}\right)\end{array}$ & $\begin{array}{c}\text { Nitrito } \\
\left(\mathrm{mg} \mathrm{L}^{-1}\right)\end{array}$ & $\begin{array}{c}\text { Condutividade } \\
\left.(\mu \mathrm{S} \mathrm{cm})^{-1}\right)\end{array}$ \\
\hline G1 & $4,84 \pm 2,34$ & $37,59 \pm 15,54$ & $29,7 \pm 2,23$ & $17,14 \pm 0,57$ & $75,27 \pm 2,75$ \\
G2 & $4,56 \pm 2,48$ & $53,03 \pm 27,66$ & $29,65 \pm 2,02$ & $21,96 \pm 2,21$ & $77,04 \pm 2,88$ \\
G3 & $3,80 \pm 2,63$ & $45,74 \pm 17,55$ & $29,36 \pm 2,13$ & $23,38 \pm 1,48$ & $7,51 \pm 0,27$ \\
\hline
\end{tabular}


ao parasitismo e maior proximidade entre os indivíduos (Brum, 2003; Belo et al., 2005).

Não foi observada diferença significativa para glicemia entre os grupos (Tabela 3). Os valores da glicemia dos peixes do grupo 3, com pesca e alta densidade, foram próximos aos encontrados por Martins et al. (2002), também em tambacus, após estímulos consecutivos de estresse de captura (115,52 mg $\left.100 \mathrm{~mL}^{-1}\right)$. Em situações de estresse, a glicemia aumenta e retorna aos níveis basais após 24 horas (Gomes et al., 2003). No presente trabalho, o sangue foi colhido 24 horas após o último episódio de pesca, e é possível que os valores dos grupos 2 e 3 já sejam valores de retorno, pois são significativamente semelhantes aos do grupo 1. Contudo, como o experimento durou três meses, os peixes podem ter perdido a sensibilidade ao estresse (Martins et al., 2002), ou seja, por "feedback" negativo aos estímulos repetidos da pesca, pode ter havido adaptação fisiológica ou resistência ao estresse.

O hematócrito foi significativamente menor nos peixes do grupo 3, em comparação aos dos grupos 1 e 2, que sofreram menor nível de agressão (Tabela 3). Resultados semelhantes foram obtidos por Tavares-Dias

Tabela 2. Valores de F, coeficiente de variação e valores médios de intensidade de pesca, ganho de peso $(\mathrm{g})$ e número de Monogenoides em tambacus dos grupos 1,2 e 3 .

\begin{tabular}{lccc}
\hline Variável & Intensidade de pesca & Ganho de peso & Parasitos \\
\hline F para grupos & $2,06^{\mathrm{ns}}$ & $38,1^{* *}$ & $6,51^{* *}$ \\
CV (\%) & 74,82 & 95,91 & 87,58 \\
Média G1 & - & $231,78 \mathrm{a}$ & $111,30 \mathrm{~b}$ \\
Média G2 & 1,54 & $106,82 \mathrm{ab}$ & $174,70 \mathrm{ab}$ \\
Média G3 & 1,83 & $62,47 \mathrm{~b}$ & $305,79 \mathrm{a}$ \\
\hline
\end{tabular}

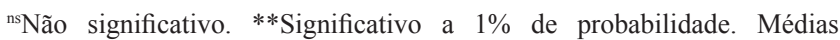
seguidas por letras distintas indicam diferença significativa pelo teste de Tukey, a 5\% de probabilidade. G1, sem pesca e baixa densidade; G2, com pesca e baixa densidade; G3, com pesca e alta densidade. et al. (2001b) em tambaqui (C. macropomum), após estímulo de captura; porém, Benfey \& Biron (2000) e Martins et al. (2000) relataram o contrário. Para os valores de eritrócitos e hemoglobina, as diferenças entre as médias não foram significativas. Tavares-Dias et al. (2001b) também constataram redução dessas duas variáveis em C. macropomum, enquanto Benfey \& Biron (2000) observaram aumento do número de eritrócitos em trutas estressadas. Assim, no presente trabalho, pode-se inferir que um quadro de hemodiluição esteja ocorrendo nos tambacus, com aumento do plasma para permitir maior fluidez para o transporte sanguíneo. Essa hemodiluição ocorre em razão da ação de catecolaminas liberadas pelo estresse que aumentam a permeabilidade dos capilares, o que causa um influxo de água para os vasos (Abreu, 2007).

O número de leucócitos varia entre família, gênero e espécie de peixe e de acordo com o ambiente em que se encontram (Tavares-Dias \& Moraes, 2004). Martins et al. (2002) demonstraram que, em situação de estresse, ocorre diminuição de linfócitos, em tambacus, com estímulos consecutivos. Já Tavares-Dias et al. (2001b), observaram o inverso em tambaquis capturados e expostos ao ar.

No presente trabalho, os valores médios não apresentaram diferença significativa entre monócitos, linfócitos, neutrófilos, leucócitos granulares PAS positivos e eosinófilos, nos três grupos. Porém, há relatos da ocorrência de neutrofilia em condições de estresse, em pacu (Martins et al., 2000) e em tambaqui (Tavares-Dias et al., 2001b). Martins et al. (2000), ao utilizar estímulo repetitivo de captura, verificaram aumento de leucócitos granulares PAS positivos e redução de monócitos em pacu. No entanto, os trombócitos aumentaram significativamente do grupo 1 para o grupo 2 e deste para o grupo 3 , de

Tabela 3. Valores de F, coeficientes de variação e valores médios das variáveis hematológicas no híbrido tambacu dos três viveiros analisados (grupos 1, 2 e 3).

\begin{tabular}{|c|c|c|c|c|c|c|c|c|c|c|}
\hline Variável & $\begin{array}{c}\text { Glicemia } \\
\left(\mathrm{mg} 100 \mathrm{~mL}^{-1}\right)\end{array}$ & $\begin{array}{l}\text { Eritrócito } \\
\left(\mathrm{x} 10^{3} \mu \mathrm{L}^{-1}\right)\end{array}$ & $\begin{array}{l}\text { Hemoglobina } \\
\left(\mathrm{g} \mathrm{dL}^{-1}\right)\end{array}$ & Hematócrito & Trombócito & Monócito & $\begin{array}{l}\text { Linfócito } \\
-(\%)--\end{array}$ & Neutrófilo & LG-PAS & Eosinófilo \\
\hline F para grupos & $1,34^{\mathrm{ns}}$ & $2,19^{\text {ns }}$ & $2,58^{\mathrm{ns}}$ & $8,05 * *$ & $5,82 * *$ & $1,65^{\text {ns }}$ & $0,49^{\text {ns }}$ & $0,82^{\text {ns }}$ & $1,36^{\mathrm{ns}}$ & $0,27^{\mathrm{ns}}$ \\
\hline $\mathrm{CV}(\%)$ & 30,85 & 9,23 & 10,02 & 9,16 & 6,30 & 96,25 & 40,38 & 171,77 & 137,85 & 94,07 \\
\hline Médias G1 & 118,52 & 3701,50 & 11,48 & $46,84 \mathrm{a}$ & $23,16 b$ & - & - & - & - & - \\
\hline Médias G2 & 114,83 & 3604,50 & 11,89 & $44,00 \mathrm{ab}$ & $24,16 \mathrm{ab}$ & - & - & - & - & - \\
\hline Médias G3 & 146,05 & 3482,00 & 11,06 & $41,65 b$ & $25,93 a$ & - & - & - & - & - \\
\hline
\end{tabular}

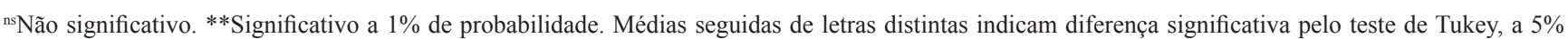
de probabilidade. G1, sem pesca e baixa densidade; G2, com pesca e baixa densidade; G3, com pesca e alta densidade. LG-PAS, leucócito granular PAS positivo. 
acordo com o aumento da agressão caracterizada pela pesca e pela alta densidade (Tabela 3). Benfey \& Biron (2000) observaram aumento na quantidade de trombócitos em resposta ao estresse agudo em trutas, enquanto Martins et al. (2000), em pacus, relataram diminuição em resposta ao estresse. A maior percentagem de trombócitos - que também são células envolvidas na defesa do organismo (Martins et al., 2009) - observada na contagem diferencial de células de defesa nos animais do grupo 3 pode estar associada à maior intensidade de parasitos neste grupo. Essa maior liberação de trombócitos na circulação também foi constatada por Garcia et al. (2007), em pacus infectados experimentalmente com Aeromonas hydrophila.

\section{Conclusões}

1. A pesca repetida, isoladamente, não afeta negativamente a fisiologia dos peixes envolvidos.

2. A sanidade dos peixes é afetada negativamente pela alta densidade populacional, o que mostra que um manejo inadequado em pesque-solte prejudica a sustentabilidade da atividade.

\section{Agradecimentos}

Ao Conselho Nacional de Desenvolvimento Científico e Tecnológico (CNPq) e à Coordenação de Aperfeiçoamento de Pessoal de Nível Superior (Capes), pela concessão de bolsas.

\section{Referências}

ABREU, J.S. Suplementação alimentar de pacu (Piaractus mesopotamicus Holmberg, 1887) com b 1,3 glicano: atividade respiratória de leucócitos, lisozima e estresse por captura. 2007. 137p. Tese (Doutorado) - Universidade Estadual Paulista, Jaboticabal.

BARTON, B.A. Stress in fishes: a diversity of responses with particular reference to changes in circulating corticosteroids. Integrative and Comparative Biology, v.42, p.517-525, 2002. DOI: $10.1093 / \mathrm{icb} / 42.3 .517$.

BELO, M.A.A.; SCHALCH, S.H.C.; MORAES, F.R.; SOARES, V.E.; OTOBONI, A.M.M.B.; MORAES, J.E.R. Effect of dietary supplementation with vitamin $\mathrm{E}$ and stocking density on macrophage recruitment and giant cell formation in the teleost fish (Piaractus mesopotamicus). Journal of Comparative Pathology, v.133, p.146-154, 2005. DOI: 10.1016/j.jcpa.2005.04.004.

BENFEY, T.J.; BIRON, M. Acute stress response in triploid rainbow trout (Oncorhynchus mykiss) and brook trout (Salvelinus fontinalis). Aquaculture, v.184, p.167-176, 2000. DOI: 10.1016/ S0044-8486(99)00314-2.

BOZZO, F.R.; MORAES, J.R.E.; MORAES, F.R. de; PEREIRA, G.T.; TAVARES-DIAS, M.; ONAKA, E.M. Kinetics of cellular component in inflammatory response induced by different stimuli in the swim bladder of pacu Piaractus mesopotamicus Holmberg, 1887 (Characidae). Journal of the World Aquaculture Society, v.38, p.302-308, 2007. DOI: 10.1111/j.1749-7345.2007.00100.x.

BROADHURST, M.K.; BARKER, D.T. Effects of capture by hook and line on plasma cortisol, scale loss and survival in juvenile mulloway, Argyrosomus hololepidotus. Archive of Fishery and Marine Research, v.48, p.1-10, 2000.

BRUM, C.D. Efeito do estresse e da suplementação alimentar com vitamina $\mathbf{C}$ sobre a formação de gigantócitos em Piaractus mesopotamicus Holmberg, 1887. 2003. 76p. Dissertação (Mestrado) - Universidade Estadual Paulista, Jaboticabal.

COOKE, S.J.; SUSKI, C.D. Do we need species-specific guidelines for catch-and-release recreational angling to effectively conserve diverse fishery resources? Biodiversity and Conservation, v.14, p.1195-1209, 2005. DOI: 10.1007/s10531-004-7845-0.

GARCIA, F.; PILARSKI, F.; ONAKA, E.M.; MORAES, F.R.; MARTINS, M.L. Hematology of Piaractus mesopotamicus fed diets supplemented with vitamins $\mathrm{C}$ and $\mathrm{E}$, challenged by Aeromonas hydrophila. Aquaculture, v.271, p.39-46, 2007. DOI: 10.1016/j.aquaculture.2007.06.021.

GEORGIADIS, M.P.; GARDNER, I.A.; HEDRICK, R.P. The role of epidemiology in the prevention, diagnosis, and control of infectious diseases of fish. Preventive Veterinary Medicine, v.48, p.287-302, 2001. DOI: 10.1016/S0167-5877(00)00202-6.

GOMES, L. de C.; ARAUJO-LIMA, C.A.R.M.; ROUBACH, R.; URBINATI, E.C. Avaliação dos efeitos da adição de sal e da densidade no transporte de tambaqui. Pesquisa Agropecuária Brasileira, v.38, p.283-290, 2003. DOI: 10.1590/ S0100-204X2003000200016.

GOMES, L. de C.; CHIPPARI-GOMES, A.R.; LOPES, N.P.; ROUBACH, R.; ARAUJO-LIMA, C.A.R.M. Efficacy of benzocaine as anesthetic in juvenile tambaqui Colossoma macropomum. Journal of the World Aquaculture Society, v.32, p.426-431, 2001. DOI: 10.1111/j.1749-7345.2001.tb00470.x.

GONÇALVES, A.C.S.; MURGAS, L.D.S.; ROSA, P.V. e; NAVARRO, R.D.; COSTA, D.V. da; TEIXEIRA, E. de A. Desempenho produtivo de tambacus alimentados com dietas suplementadas com vitamina E. Pesquisa Agropecuária Brasileira, v.45, p.1005-1011, 2010. DOI: 10.1590/ S0100-204X2010000900010.

IWAMA, G.K.; AFONSO, L.O.B.; TODGHAM, A.; ACKERMAN, P.; NAKANO, K. Are hsps suitable for indicating stressed states in fish. The Journal of Experimental Biology, v.207, p.15-19, 2004. DOI: $10.1242 /$ jeb.00707.

JORDÃO, L.C.; VOLPATO, G.L. Chemical transfer of warming information in non-injured fish. Behaviour, v.137, p.681-690, 2000. DOI: $10.1163 / 156853900502286$.

KUBITZA, F. Tilápia: tecnologia e planejamento na produção comercial. 2.ed. Jundiaí: DEGSPARI, 2011. 316p. 
MARENGONI, N.G.; SANTOS, R.S.; GONÇALVES JÚNIOR, A.C.; GINO, D.M.; ZERBINATTI, D.C.P.; LIMA, F.S. Monogenoidea (Dactylogyridae) em tilápias-do-nilo cultivadas sob diferentes densidades de estocagem em tanques-rede. Arquivo Brasileiro de Medicina Veterinária e Zootecnia, v.61, p.393-400, 2009. DOI: 10.1590/S0102-09352009000200016.

MARTINS, M.L.; MORAES, F.R. de; FUJIMOTO, R.Y.; NOMURA, D.T.; FENERICK JUNIOR, J. Respostas do híbrido tambacu (Piaractus mesopotamicus Holmberg, 1887, macho $\mathrm{x}$ Colossoma macropomum Cuvier, 1818, fêmea) a estímulos simples e consecutivos de captura. Boletim do Instituto de Pesca, v.28, p.195-204, 2002.

MARTINS, M.L.; MORAES, F.R. de; FUJIMOTO, R.Y.; ONAKA, E.M.; BOZZO, F.R.; MORAES, J.R.E. de. Carrageenin induced inflammation in Piaractus mesopotamicus (Osteichthyes: Characidae) cultured in Brazil. Boletim do Instituto de Pesca, v.32, p.31-39, 2006.

MARTINS, M.L.; MORAES, F.R. de; MORAES, J.R.E. de; MALHEIROS, E.B. Falha na resposta do cortisol ao estresse por captura e por carragenina em Piaractus mesopotamicus Holmberg, 1887 (Osteichthyes: Characidae). Acta Scientiarum. Biological Sciences, v.22, p.545-552, 2000.

MARTINS, M.L.; MYIAZAKI, D.M.Y.; TAVARES-DIAS, M.; FENERICK JUNIOR, J.; ONAKA, E.M.; BOZZO, F.R.; FUJIMOTO, R.Y.; MORAES, F.R. Characterization of the acute inflammatory response in the hybrid tambacu (Piaractus mesopotamicus male $\mathrm{x}$ Colossoma macropomum female) (Osteichthyes). Brazilian Journal of Biology, v.69, p.631-637, 2009. DOI: 10.1590/S1519-69842009000400026.

OBA, E.T.; MARIANO, W. dos S.; SANTOS, L.R.B. dos. Estresse em peixes cultivados: agravantes e atenuantes para o manejo rentável. In: TAVARES-DIAS, M. (Org.). Manejo e sanidade de peixes em cultivo. Macapá: Embrapa Amapá, 2009. p.226-247.
PAPOUTSOGLOU, S.E.; TZIHA, G.; VRETTOS, X.; ATHANASIOU, A. Effects of stocking density on behaviour and growth rate of European sea bass (Dicentrarchus labrax) juveniles reared in a close circulated system. Aquacultural Engineering, v.18, p.135-144, 1998. DOI: 10.1016/S0144-8609(98)00027-2.

TAVARES-DIAS, M.; MARTINS, M.L.; MORAES, F.R. Fauna parasitária de peixes oriundos de "pesque-pague" do município de Franca, São Paulo, Brasil. I. Protozoários. Revista Brasileira de Zoologia, v.18, p.67-79, 2001a. DOI: 10.1590/ S0101-81752001000500005.

TAVARES-DIAS, M.; MORAES, F.R. de. Hematologia de peixes teleósteos. Ribeirão Preto: Villimpress, 2004. 144p.

TAVARES-DIAS, M.; ONO, E.A.; PILARSKI, F.; MORAES, F.R. Can thrombocytes participate in the removal of cellular debris in the blood circulation of teleost fish? A cytochemical study and ultrastructural analysis. Journal of Applied Ichthyology, v.23, p.709-712, 2007. DOI: 10.1111/j.1439-0426.2007.00850.x.

TAVARES-DIAS, M.; SANDRIM, E.F. da S.; MORAES, F.R. de; CARNEIRO, P.C.F. Physiological responses of "tambaqui" Colossoma macropomum (Characidae) to acute stress. Boletim do Instituto de Pesca, v.27, p.43-48, $2001 \mathrm{~b}$.

VOLPATO, G.L. Pesque-e-solte: uma análise crítica. Plural, v.1, p.9-20, 2000.

WISENDEN, B.D.; CHIVERS, D.P. The role of public chemical information in antipredator behaviour. In: LADICH, F.; COLLIN, S.P.; MOLLER, P.; KAPOOR, B.G. Communication in fishes. Enfield: Science Publishers, 2006. p.259-278.

WISENDEN, B.D.; STACEY, N.E. Fish semiochemicals and the evolution of communication networks. In: MCGREGOR, P.K. (Ed.). Animal communication networks. Cambridge: Cambridge University, 2005. p.540-567. DOI: 10.1017/ CBO9780511610363.028.

Recebido em 30 de junho de 2011 e aprovado em 19 de novembro de 2012

Pesq. agropec. bras., Brasília, v.48, n.8, p.1058-1063, ago. 2013 DOI: 10.1590/S0100-204X2013000800035 